\title{
"Riesgos ergonómicos relacionados a la lumbalgia ocupacional en enfermeras que laboran en Centro Quirúrgico del Hospital Daniel Alcides Carrión, 2014"
}

\author{
"Ergonomic risks related to occupational low back pain in nurses who work in Center Surgical Hospital \\ Daniel Alcides Carrión, 2014"
}

Milagros Guizado Ramos ${ }^{1}$, Karin Zamora Cordova ${ }^{2}$

\section{RESUMEN}

Objetivo: Determinar la relación que existe entre los riesgos ergonómicos y la lumbalgia ocupacional en enfermeras que laboran en Centro Quirúrgico del Hospital Daniel Alcides Carrión, 2014. Materiales y Métodos: La investigación es aplicada al campo de la salud, tipo descriptivo correlacional, de corte transversal, no experimental, muestreo no probabilístico de carácter intencional. Muestra conformada por 33 enfermeras; nombradas $(72.7 \%)$ y contratadas $(27.3 \%)$, con edades comprendidas entre 23 a 33 años (69.70\%). Resultados: Existe una "correlación moderada media positiva" (Rho de Spearman 0.517) entre los riesgos ergonómicos (posturas forzadas prolongadas (0.718) y movimientos corporales $(0.649)$ ambas con un nivel de correlación alta positiva) con la lumbalgia ocupacional en la dimensión aguda, y los riesgos peso fuerza (0.619), movimientos corporales $(0.603)$ y posturas forzadas prolongadas $(0.436)$ presentan una "correlación moderada media y alta positiva" respectivamente, tienen un mayor nivel de relación con la lumbalgia sub aguda y la postura corporal (0.055), peso fuerza (0.158) ambas con una "correlación baja positiva", no presentan un mayor nivel de relación con la lumbalgia crónica. Conclusion: Los riesgos ergonómicos se relacionan con la lumbalgia ocupacional, con una probabilidad menor de $\mathrm{P}(0,02)$ y un nivel de correlacion moderada media positiva aplicando el estadístico de Rho de Spearman 0.517.

Palabras Clave: Riesgos ergonómicos, Lumbalgia ocupacional, lumbalgia aguda, sub aguda y crónica.

\begin{abstract}
Objective: To determine the relationship between ergonomic hazards and occupational low back pain in nurses working in Surgical Center Hospital Daniel Alcides Carrión, 2014. Materials and Methods: The research is applied to the field of health, correlational descriptive, cutting transversal and not experimental, non-probabilistic intentional sampling. Sample consisted of 33 nurses; named (72.7\%) and contracted (27.3\%), aged 23-33 years (69.70\%). Results: With a lower probability of $\mathrm{P}(0.02)$ there is a "positive average moderate correlation" (Spearman rho 0.517) between the ergonomic risks (prolonged stress positions $(0.718)$ and body movements (0.649) both with a level of correlation positive) high with occupational low back pain in acute dimension, and risk weight force (0.619), body movements $(0.603)$ and forced postures prolonged (0.436) have an "average positive moderate correlation and high" respectively, have a higher level of relationship with subacute low back pain and posture $(0.055)$, weight force $(0.158)$ both with a "low positive correlation" does not have a higher level of relationship with chronic low back pain. Conclusion: Ergonomic hazards related to occupational low back pain, with a lower probability of $\mathrm{P}(0.02)$ and a moderate level of average positive correlation using the Spearman rho statistic 0.517.
\end{abstract}

Key words: Ergonomic hazards, occupational low back pain, acute low back pain, acute and chronic sub.

\footnotetext{
${ }^{1}$ Universidad Privada Norbert Wiener

${ }^{2}$ Universidad Privada Norbert Wiener
}

\section{INTRODUCCION}

La Organización Mundial de la Salud en el 2011, estima que en América Latina y el Caribe la notificación de enfermedades ocupacionales alcanza el $1 \%$ y el $5 \%$ de los casos, ya que, por lo general, se registra solamente los casos que causan incapacidad sujeta a indemnización (1). En América Latina y el Perú aún no se conoce bien la magnitud que alcanzan las enfermedades ocupacionales. A pesar de que se está investigando y promoviendo la Salud Ocupacional, con 
INVESTIGACIÓN ORIGINAL ORIGINAL RESEARCH
Riesgos ergonómicos relacionados a la lumbalgia ocupacional en enfermeras que laboran en Centro Quirúrgico del Hospital Daniel Alcides Carrión - 2014. frecuencia los trabajadores están expuestos a riesgos físicos, químicos, biológicos, psicosociales $\mathrm{y}$ ergonómicos presentes en las actividades laborales. Dichos riesgos pueden conducir a una ruptura del estado de salud y pueden causar accidentes, enfermedades ocupacionales y otras relacionadas con el ambiente laboral (2).

El dolor de espalda representa un importante problema de salud pública en las sociedades occidentales por su alta prevalencia y repercusión socioeconómica: genera un alto número de consultas a diferentes profesionales, una elevada utilización de los servicios sanitarios y una considerable pérdida de días de trabajo. ${ }^{(5)}$

La prevalencia anual del dolor lumbar, en población general, oscila según diferentes estudios entre el $22 \%$ y $65 \%$. (3) Por otro lado, se estima que la prevalencia de dolor lumbar crónico es del $7,5 \%$ en mujeres (95\% $\mathrm{IC}: 7,0-8,1)$ y del 7,9\% en hombres (95\% Intervalo de Confianza (IC): 7,2$8,5)$.

La lumbalgia ocasiona importantes costos fundamentalmente derivados de la Incapacidad Temporal (IT). El 11,4\% del total de las IT que se tramitaron durante el año 1997 en España se debe a dolor lumbar. El riesgo laboral de enfermeras en centro quirúrgico es un tema que cada vez cobra mayor importancia. Toda vez que existe estudios que demuestran la existencia de la exposición de riesgos ergonómicos (4).

Actualmente se reconoce que la prevención de riesgos ergonómicos es la base para una gestión activa de la seguridad y salud en el trabajo, es por ello que se debe planificar una acción preventiva a partir de la identificación de riesgos ocupacionales, evaluar los riesgos a la hora de elegir los equipos de trabajo, sustancias o preparados químicos y el acondicionamiento de lugares de trabajo y controlarlo cuando superen los límites permisibles $\mathrm{y}$ así disminuir los riesgos ergonómicos (5).

El personal de enfermería es un miembro imprescindible dentro del grupo multidisciplinario por la función que desempeña constantemente en sala de operaciones como enfermera instrumentista y enfermera circulante. La cual es brindar seguridad y eficiencia. En el Centro Quirúrgico del Hospital Daniel Alcides Carrión, se observa que algunas enfermeras presentan cefaleas, problemas respiratorios, lumbalgias, varices en miembros inferiores, entre otros. Al interactuar refieren que todo el día están de pie, que cargan cajas pesadas de instrumental, a veces tienen que pasar a pacientes obesos de la mesa quirúrgica a la camilla y que el olor del glutaraldehido les produce a veces cefaleas entre otras expresiones (6).

En nuestro país se realizan pocos estudios de investigación sobre riesgos ocupacionales en el sector salud, además son las empresas privadas quienes ponen énfasis en la salud ocupacional de sus trabajadores, ya que estos pasan exámenes de ingreso, anuales y al término de sus trabajos mientras que las entidades del estado, se toma poco interés en evaluar la salud ocupacional de sus trabajadores, ni siquiera cuentan con un área de salud ocupacional en la mayoría de los casos. En el Hospital Nacional Daniel Alcides Carrión, se ha observado que el personal de enfermería del área de Sala de Operaciones refieren de múltiples exposiciones a riesgos laborales y posibles repercusiones en su salud física, de aquí nace nuestro interés por realizar este estudio de investigación. Por la realidad anteriormente expuesta es que se decide determinar los riesgos ergonómicos relacionados con la lumbalgia en el trabajo de enfermería en el servicio de Centro Quirúrgico del Hospital Nacional Daniel Alcides Carrión.

\section{MATERIALES Y METODOS}

\section{Tipo de Investigación:}

El presente estudio es de enfoque cuantitativo de tipo correlacional, de corte transversal, pues se obtendrá información en una sola oportunidad (7).

\section{Ámbito de investigación:}

El estudio de investigación se realizó en el servicio de Centro Quirúrgico del Hospital Nacional Daniel Alcides Carrión en la Provincia Constitucional del Callao. Que brinda atención de lunes a domingo durante las 24 horas del día.

\section{Población y Muestra}

Conformada por el personal de enfermería que labora en Centro Quirúrgico del Hospital Nacional Daniel Alcides Carrión.

$$
\begin{gathered}
\text { POBLACION DEL PERSONAL DE ENFERMERIA QUE LABORA EN } \\
\text { CENTROQUTRURGICO DEL HOSPTAL NACIONAL DANEL } \\
\text { ALCIDES CARRIÓN }
\end{gathered}
$$

$\begin{array}{lll}\text { PERSONAL NOMBRADO } & 24 & 72.7 \% \\ \text { PERSONAL CAS } & 9 & 27.3 \% \\ \text { TOTAL } & 33 & 100 \%\end{array}$


INVESTIGACIÓN ORIGINAL ORIGINAL RESEARCH
Riesgos ergonómicos relacionados a la lumbalgia ocupacional en enfermeras que laboran en Centro Quirúrgico del Hospital Daniel Alcides Carrión - 2014.
La muestra equivale a la totalidad de la población, por ser importante para el estudio establecer la relación de las variables en estudio en todo el personal de enfermería. Muestreo, se obtuvo de manera no probabilística, mediante una selección intencional, de acuerdo a los criterios de inclusión y exclusión.

Los criterios de inclusión son: Enfermeros (as) que se encuentran laborando en Centro Quirúrgico del Hospital Nacional Daniel Alcides Carrión. Enfermeros (as) que registran descansos médicos por problemas musculo esqueléticos en el área de personal del HNDAC. Enfermeros (as) que acepten participar en el estudio.

Los criterios de exclusión son: Enfermeros que se encuentren de vacaciones, con licencia por enfermedad o embarazo. Enfermera con cargo administrativo. Enfermeros que no completen el instrumento. Enfermeras que no registran permisos por descanso médico por problemas musculo esqueléticos en el área de personal del HNDAC.

\section{Técnicas e instrumentos de recolección de datos}

Para evaluar los riesgos ergonómicos se elaboró un cuestionario. Para medir la lumbalgia ocupacional se elaboró un instrumento con 32 preguntas que teniendo en consideración la parte teórica de tiempo de evolución de la lumbalgia se clasifica en aguda, sub-aguda y crónica, de manera fiable según el grado de incapacidad física derivado de la lumbalgia. A este respecto, la "incapacidad física" se define como la limitación en la realización de las actividades cotidianas en diverso grado de intensidad.

El cuestionario es de 32 preguntas considerándose como alternativas

- nunca-1

- Algunas veces-2

- Frecuentemente-3

- Siempre-4

\section{Validación del instrumento:}

Para determinar la validez del instrumento del presente trabajo de investigación, se sometió al "juicio de experto", para lo cual 04 especialistas en salud ocupacional con grado de Maestro y Doctor validaron y aprobaron el instrumento de recolección de datos.

La confiabilidad del instrumento; se determinó con el coeficiente del Alfa de Cronbach. La confiabilidad, se refiere a la consistencia o estabilidad de una medida. Una definición técnica de confiabilidad es aquella que parte de la investigación de qué tanto error existe en un instrumento de medición. Dependiendo del grado en que los errores de medición estén presentes en un instrumento de medición, el instrumento será poco o más confiable (6).

Para verificar la confiabilidad del instrumento de evaluación a ser empleado, se aplicó el coeficiente Alfa de Cronbach a las 32 preguntas, con el apoyo del software IBM SPSS Statistics versión 23 teniendo como resultado el valor de "alfa" $(\alpha)$ de 0,825 .

\section{Plan de procesamiento y análisis de datos}

Los datos recolectados fueron codificados y digitados mediante el programa Microsoft Excel 2010. Los datos fueron exportados al software estadístico SPSS Versión 23.0 obteniendo como producto la base de datos de la investigación. Para la realización de un análisis estadístico descriptivo, se utilizo tablas de contingencia y gráficos correspondientes que permitieron describir la información del estudio.

Para la realización del contraste de hipótesis, se eligió el coeficiente de correlación de rangos de Spearman ó Rho de Spearman, aceptando valores p menor 0,05 como significativo.

Este coeficiente es una prueba estadística no paramétrica que mide la correlación entre dos variables. Para calcular $\boldsymbol{\rho}$, los datos son ordenados y reemplazados por su respectivo orden. El estadístico $\boldsymbol{\rho}$ viene dado por la expresión.

$$
\rho=1-\frac{6 \Sigma \nu^{2}}{N\left(N^{2}-1\right)}
$$

El valor del coeficiente de correlación de Spearman oscila entre menos uno (-1) y más uno $(+1)$, indicándonos asociaciones negativas o positivas respectivamente. El nivel de correlación se determinó comparando el resultado obtenido con la siguiente tabla de correlación:

Aspectos éticos: Se obtuvo el consentimiento informado de los usuarios participantes.

\section{RESULTADOS}

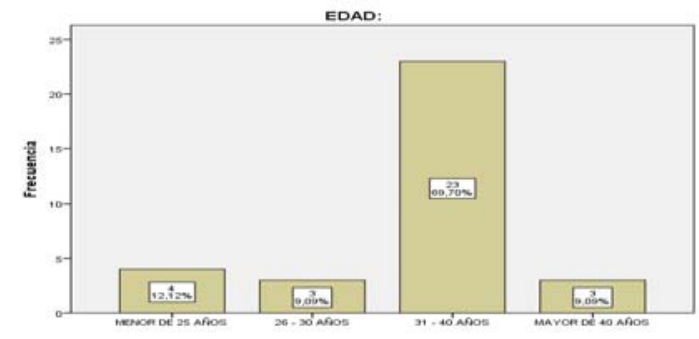

Grafico 1: Características de la población en estudio 
INVESTIGACIÓN ORIGINAL ORIGINAL RESEARCH
Riesgos ergonómicos relacionados a la lumbalgia ocupacional en enfermeras que laboran en Centro Quirúrgico del Hospital Daniel Alcides Carrión - 2014.
Interpretación: Se observa en el gráfico 1, un $69.70 \%$ que son 23 de 33 enfermeras que sus edades se encuentran entre 31 a 40 años, frente a un $12.12 \%$ (4/33) que tienen edades menores de 25 años y en igual medida un $9.09 \%$ que corresponde a 3/33 enfermeras que tiene entre 26 a 30 años y mayores a 40 respectivamente.

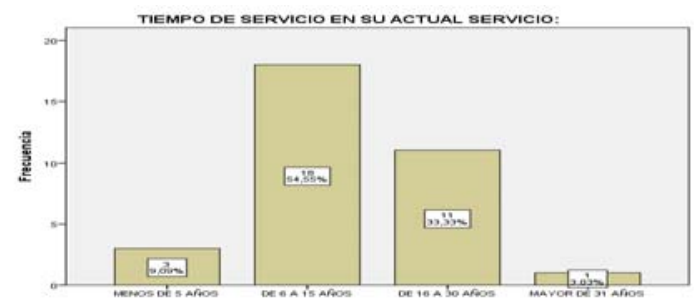

Grafico 2:

Interpretación: Se observa en el gráfico 2, un $54.55 \%$ corresponde a 18 de 33 enfermeras que se encuentran laborando entre 6 a 15 años, frente a un $33.33 \%$ (11/33) que tienen laborando entre 16 a 30 años y un $9.09 \%(3 / 33)$ enfermeras que tienen menos de 5 años laborando en el servicio de centro quirúrgico del Hospital DAC.

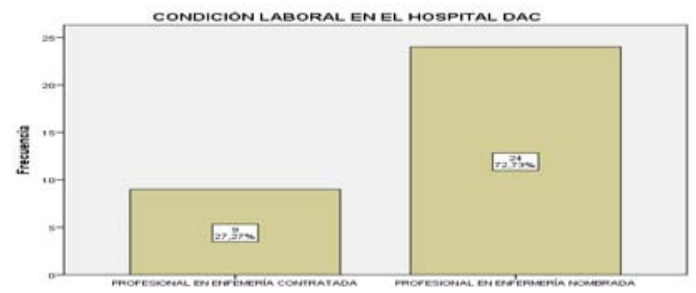

Grafico 3:

Interpretación: Se observa en el gráfico 3, un $72.73 \%$ corresponde a 24 de 33 enfermeras que se encuentran en la condición laboral de nombradas frente a un $27.27 \%$ que lo conforman 9 de 33 enfermeras que son de condición contratadas (CAS).

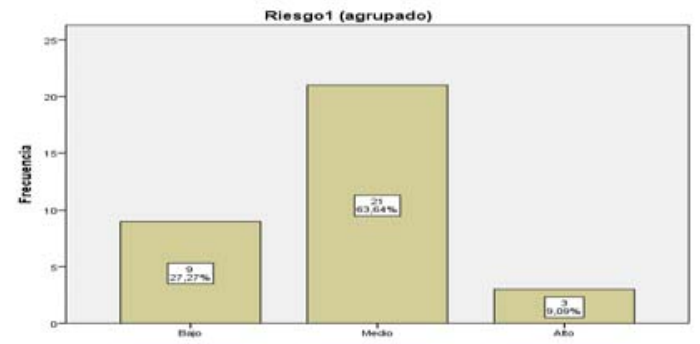

Grafico 4: Riesgos ergonomicos (movimientos corporales,postura corporal, peso_fuerza, posturas forzadas prolongadas, confort) agrupados según niveles de riesgo.
Interpretación: En el grafico 4, se observa que los riesgos ergonómicos agrupados (las cinco dimensiones: movimienos corporales,postura corporal, peso fuerza, posturas forzadas prolongadas, confort) se encuentran en un riesgo nivel bajo; porque 9 de 33 enfermeras presentan lumbalgia sub aguda (6/9) y crónica (3/9), en cambio en un riesgo nivel medio; hay 21 de 33 enfermeras que presentan lumbalgia aguda (3/21), sub aguda (17/21) y crónica $(1 / 21)$, finalmente en riesgo nivel alto; se encuentran solo 3 de las 33 enfermeras presentando lumbalgia aguda $(2 / 3)$ y sub aguda $(1 / 3)$.

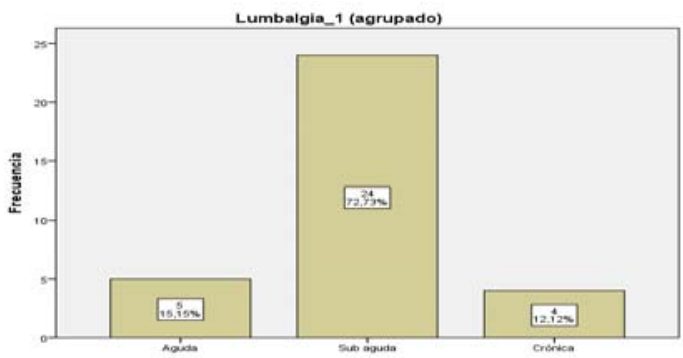

Grafico 5: Lumbalgia agrupada (según dimensiones: aguda, subaguda y crónica)

Interpretación: En el grafico 5, se observa que la lumbalgia ocupacional agrupada (se ha clasificado en tres niveles según el tiempo de evolución: aguda ( $>4$ semanas); sub_aguda (entre 4 a 12 semanas) crónica $(<12$ semanas)) asi se ha encontrado que 5 de 33 enfermeras están clasificadas en lumbalgia ocupacional aguda (15.2\%), asi también 24 de 33 enfermeras tienen lumbalgia ocupacional sub_aguda $(72.7 \%)$ y solo 4 de 33 enfermeras se encuentran clasificadas en lumbalgia ocupacional crónica (12.1\%).

Tabla 1: Correlaciones entre los riesgos ergonomicos con la lumbalgia ocupacional

\begin{tabular}{|c|c|c|c|c|}
\hline \multirow{2}{*}{$\begin{array}{c}\text { RIESGOS } \\
\text { ERGONOMICOS }\end{array}$} & \multicolumn{4}{|c|}{ LUMBALGIA OCUPACIONAL } \\
\hline & & AGUDA & SUB_AGUDA & CRONICA \\
\hline MOVIMIENTOS & Rho de Spearman & 0.649 & 0.603 & -0.115 \\
\hline \multicolumn{5}{|l|}{ CORPORALES } \\
\hline \multirow{3}{*}{ POSTURA CORPORAL } & Sig. & 0.407 & 0.256 & 0.523 \\
\hline & Rho de Spearman & 0.263 & 0.277 & 0.055 \\
\hline & Sig. & 0.364 & 0.323 & 0.762 \\
\hline \multirow[t]{2}{*}{ PESO_FUERZA } & Rho de Spearman & 0.826 & 0.619 & 0.158 \\
\hline & Sig. & 0.483 & 0.916 & 0.379 \\
\hline POSTURAS_FORZADAS & Rho de Speaman & 0.718 & 0.436 & -0.375 \\
\hline _PROLONGADAS & Sig. & 0.921 & 0.451 & 0.032 \\
\hline \multirow{2}{*}{ CONPORT } & Rho de Spearman & -0.035 & -0.299 & -0.501 \\
\hline & Sig. & 0.846 & 0.091 & 0.003 \\
\hline
\end{tabular}


INVESTIGACIÓN ORIGINAL ORIGINAL RESEARCH
Riesgos ergonómicos relacionados a la lumbalgia ocupacional en enfermeras que laboran en Centro Quirúrgico del Hospital Daniel Alcides Carrión - 2014.
En la tabla 1, se observa que con un menor nivel de significación de uno por ciento (1\%); se rechaza la hipótesis nula (Ho) y se acepta la hipótesis alterna (Ha1). Se concluye que tienen mayor nivel de relación con la lumbalgia aguda las dimensiones peso/fuerza (0.826) "correlacion intensa positiva" seguida de posturas/forzadas/prolongadas (0.718) "correlacion alta positiva", seguida de los movimientos corporales (0.649) con una "correlacion moderada alta positiva", asi la postura corporal (0.263) expresa una "correlacion moderada baja positiva", y finalmente la dimension confort $(-0.035)$ con una "correlacion moderada baja inversa". Asi mismo que tienen mayor nivel de relación con la lumbalgia sub_aguda las dimensiones peso/fuerza (0.619) y movimientos corporales (0.603) que expresan igualmente una "correlacion moderada alta positiva" y posturas/forzadas/prolongadas $(0.436)$ una "correlacion moderada media positiva", seguida de la postura corporal $(0.277)$ con una "correlacion moderada baja positiva", y finalmente la dimension confort (-0.299) con una "correlacion baja inversa".

Finalmente que tienen mayor nivel de relación con la lumbalgia cronica las dimensiones postura corporal $(0.055)$ y peso/fuerza $(0.158)$ ambas con una "correlacion baja positiva" seguida de la dimensión posturas/forzadas/prolongadas(-0.375) una "correlacion moderada baja inversa" y movimientos corporales $(-0.115)$ con una "correlacion baja inversa" y finalmente la dimension confort $(-0.501)$ con una "correlacion moderada media inversa".

\section{Contrastacion de la hipotesis estadística}

\section{Hipotesis nula (Ho)}

Los riesgos ergonómicos no estan relacionados significativamente con la lumbalgia ocupacional en enfermeras que laboran en centro quirúrgico del hospital nacional daniel alcides carrión, 2014.

\section{Hipotesis alterna (Ha)}

Los riesgos ergonómicos estan relacionados significativamente con la lumbalgia ocupacional en enfermeras que laboran en centro quirúrgico del hospital nacional daniel alcides carrión, 2014.

\begin{tabular}{|c|c|c|c|c|}
\hline & & & $\begin{array}{c}\text { Riesgo1 } \\
\text { (agupado) }\end{array}$ & $\begin{array}{l}\text { Lumbalgia } \\
\text { (agupad }\end{array}$ \\
\hline \multirow[t]{5}{*}{$\begin{array}{l}\text { Rho de } \\
\text { Spearman }\end{array}$} & $\begin{array}{l}\text { Riesgo1 } \\
\text { (agrupado) }\end{array}$ & $\begin{array}{l}\text { Coeficiente de } \\
\text { correlación } \\
\text { Sig. (bilater al) }\end{array}$ & 1,000 & \\
\hline & & $\mathrm{N}$ & 33 & \\
\hline & $\begin{array}{l}\text { Lumbalgia_1 } \\
\text { (agrupado) }\end{array}$ & $\begin{array}{l}\text { Coeficiente de } \\
\text { correlación }\end{array}$ & $.517^{-}$ & \\
\hline & & Sig. (bilater al) & .002 & \\
\hline & 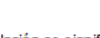 & $\mathrm{N}$ & 33 & \\
\hline
\end{tabular}

En la tabla 2, se evidencia con una probabilidad de $\mathrm{p}(0,02)$ menor al nivel de significación de uno por ciento (1\%); que se rechaza la hipótesis nula (Ho) y se acepta la hipótesis alterna (Ha).

Por tanto, se concluye que existe una relación significativa entre la variable riesgos ergonomicos y la variable lumbalgia ocupacional; cuyo Coeficiente de Correlación "Rho de Spearman" es 0,517 que expresa una "correlacion moderada media positiva".

\section{DISCUSIÓN}

Los riesgos ergonómicos según los resultados del estudio se relacionan con la lumbalgia ocupacional considerando una probabilidad menor de $\mathrm{P}(0,02)$ con un nivel de correlacion moderada media positiva aplicando el estadístico de Rho de Spearman 0.517 .

En las hipótesis especificas se tiene como resultado que hay mayor nivel de relación con la lumbalgia aguda las dimensiones peso/fuerza $(0.826)$ con un nivel de correlacion intensa positiva ; asi las posturas/forzadas/prolongadas (0.718) y movimientos corporales (0.649) ambas con un nivel de correlacion alta positiva, y la postura corporal (0.263) con un nivel de correlacion moderada baja positiva.

Los resultados también enfatizan que tienen mayor nivel de relación con la lumbalgia sub_aguda las dimensiones peso/fuerza (0.619), la dimensión movimientos corporales $(0.603)$ y la dimensión posturas/forzadas/prolongadas (0.436) con niveles de correlacion moderada media positiva y moderada alta positiva respectivamente, seguida de la dimensión postura corporal $(0.277)$ con un nivel de correlacion moderada baja positiva.

Los resultados indican que tienen mayor nivel de relacion con la lumbalgia cronica las dimensiones postura corporal (0.055) y la dimensión peso fuerza (0.158) ambas con un nivel de correlacion baja positiva seguida de la dimensión posturas/forzadas/prolongadas (-0.375) y movimientos corporales (-0.115) ambas con un nivel de correlacion moderada inversa y moderada baja inversa respectivamente y finalmente la dimension confort $(-0.501)$ con un nivel de correlacion moderada media inversa.

Dichos resultados se asemejan al estudio de Jimenez que en el 2011 realizo un estudio sobre investigaciòn y evaluaciòn del riesgo ergonómico orientado a las posiciones y posturologia, al cual están expuestos las enfermeras del Instituto de 
INVESTIGACIÓN ORIGINAL ORIGINAL RESEARCH
Riesgos ergonómicos relacionados a la lumbalgia ocupacional en enfermeras que laboran en Centro Quirúrgico del Hospital Daniel Alcides Carrión - 2014.
Biomecànica de Valencia" (7), en el cual los resultados obtenidos por el método E-Lest, que la mayoría de las lesiones musculo esqueléticos ocurren por técnicas incorrectas de manejo de cargas $(35.7 \%)$, y otros factores de riesgo como como las vibraciones $(12.8 \%)$, ruido $(17.6 \%)$, asi como también en el estudio de Garcia, Viteri que en 2011 realizo el estudio sobre ergonomia del puesto de trabajo en el personal de enfermería del servicio de centro quirúrgico de la Clinica de la Universidad de Navarra" (8), que indica que los monitores que se emplean en el área de cirugía en la estación de trabajo se encuentra en inadecuada posición e iluminación (24.8\%), y la temperatura y la ventilación como factor de riesgo muestra grandes inconvenientes en su funcionamiento $(28.6 \%)$, además del ruido como factor silencioso $(10.3 \%)$, la posición adoptada por tiempos prolongados ante la estación de trabajo (21.9\%).

La investigación de Declos en el año 2011, sobre Identificación de los riesgos ergonomicos laborales asociados a enfermedades sospechosas de posible origen laboral atendida en el Sistema Nacional de Salud" en España (9), refiere que efectivamente existen evidencias comprobadas de 26 enfermedades que se asocian a los factores de riesgo ergonómicos, resaltando la cervicalgia $(28.5 \%$ ), lumbalgia (alta $33.3 \%$ ), patología de dolor en la columna dorsal $(37.5 \%)$, patología de tendinitis de bíceps (61.5\%). El estudio de Faustino en el 2011, en su estudio sobre riesgo laboral en centro quirúrgico (10), indica que los factores de riesgo se presentan en el manejo de cargas (18.3\%), pesos inadecuados (17.4\%) ubicación de los equipos de trabajo (15.7\%), hay algo interesante que resaltar en el estudio de Valdez, con relación al aumento progresivo con la edad, a diferencia del estudio de Figueroa que en el 2015 en su estudio sobre riesgo ergonómico y lumbalgia ocupacional en enfermeras de centro quirúrgico del Hospital General de México" (11). que fueron los movimientos repetitivos como antecedentes de lumbalgia positiva $(47.27 \%)$ y manipulación inadecuada de cargas como antecedente de lumbalgia negativa (52.72\%).

Estudios como Alarcon que en el 2013 realizo el estudio sobre ergonomia y la relación con los factores de riesgo en salud ocupacional en el Hospital Belen" (12) el estudio evalúa a través del análisis documental las capacidades humanas tales como fuerza, postura y repeticiones de movimientos, y los aspectos psicológicos como la carga mental y la toma de decisiones, asociándose grandes fuerzas con riesgo de lesiones en el hombro $(26.67 \%)$, en la espalda baja $(40 \%)$, y el antebrazo (16.67\%), muñeca y mano (16.67\%), la relación entre la fuerza y el grado de riesgo (alto,medio, bajo) de lesión se modifica por otros factores de riesgo como la postura $(30 \%)$, la aceleración $(23.33 \%)$, la repetición $(40 \%)$, y la duración (6.67\%), el estudio de Salazar del 2011 sobre conocimiento y actitudes sobre riesgo laboral en las enfermeras del centro quirúrgico del hospital nacional Cayetano Heredia" (13), concluye en un hecho muy relevante indicando que el personal de centro quirúrgico tiene además de la labor encomendada por sus funciones colaborar en el traslado de los pacientes sin tomar a veces los cuidados necesarios, exponiéndose a sufrir daños físico y posturales, que desencadenan la lumbalgia en un nivel medio (3/33) y alto $(2 / 33)$ no representando un grado de asociación determinante en un $15.15 \%$ con la lumbalgia ocupacional aguda.

\section{CONCLUSION:}

Los riesgos ergonómicos se relacionan con la lumbalgia ocupacional, con una probabilidad menor de $\mathrm{P}(0,02)$ y un nivel de correlacion moderada media positiva aplicando el estadístico de Rho de Spearman 0.517.

\section{CORRESPONDENCIA:}

Milagros Guizado Ramos. Universidad Privada Norbert Wiener .

Avenida Arequipa 440-Santa Beatriz-Lima. Teléfono: 01-706-5100

E-mail:https://pe.linkedin.com/in/milagrosguizado-ramos-3127539b

\section{REFERENCIAS BIBLIOGRAFICAS}

1. MINISTERIO DE SALUD "Implementación del Sistema Nacional de Vigilancia de Salud Ocupacional” Lima Perú 2006. Pág.1

2. MINISTERIO DE SALUD "Manual de salud Ocupacional”. Lima- Perú 2005. Pág. 7, 24, 25,44.

3. MINISTERIO DE SALUD "Implementación del Sistema Nacional de Vigilancia de Salud Ocupacional” Lima Perú 2006. Pág.1

4. ROMERO AISMARA BORGES "Personal de enfermería, condiciones de trabajo de Alto Riesgo "Venezuela $-2^{\circ}$ edición 2003.Pag.113 - 118.

5. RUBIO, S. LUCEÑO, L. MARTIN, J Y JAEN, M. "Modelos y procedimientos de evaluación de la carga mental de trabajo". Edupsykhe. 2010. Pág. 85-108.

6. HERNÁNDEZ SAMPIERI, ROBERTO Y OTROS. "Metodología de 
INVESTIGACIÓN ORIGINAL ORIGINAL RESEARCH investigación”, México, 6 ta Edición, 2014.

7. JIMENEZ ROSERO, C, (2011) "Investigación y Evaluación del riesgo ergonómico orientado a las posiciones y posturologia, a al cual están expuestos las enfermeras del Instituto de Biomecánica de Valencia” España.

8. GARCIA, C., VITERI-RAMIREZ C., (2011) "Ergonomía del puesto de trabajo en el personal de enfermería del servicio de centro quirúrgico de la Clínica de la Universidad de Navarra" España.

9. DELCLOS, J., ALARCON, M., CASANOVAS, A., (2011) "Identificación de los riesgos ergonómicos laborales asociados a enfermedades sospechosas de posible origen laboral atendida en el Sistema Nacional de Salud", Cataluña, España.

10. FAUSTINO, M. AMELIA (2011) "nivel de conocimiento y actitudes sobre riesgo laboral en las enfermeras del centro quirúrgico del hospital nacional Cayetano Heredia”Lima. Perú.
11. ALLEMANT VALEUCIA, BÁRBARA, AGUIRRE ASENCIOS, ELIZABETH (2011) "Factores de riesgo que influyen en la salud ocupacional de los trabajadores de la Unidad de servicios centro quirúrgico del hospital Nacional Daniel Alcides Carrión Abril-Julio".

12. ALARCON GUILLEN, M., FONSECA. (2013), "Ergonomía y la relación con los factores de riesgo en salud ocupacional en el Hospital Belén" - Lambayeque.

13. SALAZAR JOAQUÍN, EMMA Y GUERRA GALÁN, DORIS FRANCISCA (2011) "conocimiento y actitudes sobre riesgo laboral en las enfermeras del centro quirúrgico del hospital nacional Cayetano Heredia" Lima. Perú.

Recibido: 21/03/2016

Aceptado: 22/06/2016 